\title{
Moderate Guidance of Improper Use of Mobile Phone by Male Nursing Students in Vocational Colleges
}

\author{
Xie Gen-tan ${ }^{1, a}$, Bi Zhi-lii, b*, Han Yu-xia ${ }^{2, c}$ \\ ${ }^{1}$ Youth League Committee, Binzhou Polytechnic, Binzhou, Shandong, China \\ ${ }^{2}$ Academy of health, Binzhou Polytechnic, Binzhou, Shandong, China \\ axiegentan@126.com \\ b*beelili@126.com \\ chanyuxia108@163.com
}

\begin{abstract}
In the study environment dominated by female students, male nursing students in higher vocational colleges will develop inappropriate behavior of using mobile phones. Excessive use of mobile phones will lead to the decline of academic performance and physical quality of male nursing students, and will affect their interpersonal communication. It is suggested that moderate guidance strategies should be adopted for male nursing students' improper use of mobile phones, and mobile phones should be turned into the most convenient learning tool by expanding students' horizons. Moderate "behavior therapy" can be used to help the male nursing students who violate the discipline to establish a new good behavior pattern. For male nursing students with serious psychological problems resulting in improper behavior of mobile phone use, psychological counseling combined with a variety of coping strategies can be adopted. This article shares a successful case of multi-party interaction to help male nursing students get rid of excessive mobile phone use.
\end{abstract}

Keywords: Vocational school, male nursing students, mobile phone use, psychological counseling, Labor Education

\section{高职男护生手机使用不当行为的适度引导}

\author{
谢根坦 ${ }^{1, a}$ 毕智丽 ${ }^{2, b *}$ 韩玉霞 ${ }^{2, c}$
}

${ }^{1}$ 滨州职业学院团委, 滨州, 山东, 中国

${ }^{2}$ 滨州职业学院健康学院, 滨州, 山东, 中国

axiegentan@126.com

b*beelili@126.com

chanyuxia108@163.com

\section{摘要}

高职男护生在女生占优势的学习环境中, 会养成手机使用的不当行为, 过度使用手机会导致男护生学习成绩和 身体素质下降，也会影响他们的人际交往。建议对男护生的手机使用不当行为采取适度引导策略，通过开拓学 生视野, 变手机为他们最便利的学习工具; 对违纪较严重的男护生可以采用适度的 “行为疗法”, 帮助他们建 立新的良好行为模式; 对由较严重心理问题导致手机使用不当行为的男护生, 可以采用心理咨询与多种方法结 合的应对策略。本文分享了一个多方联动成功帮助男护生摆脱手机过度使用行为的案例。

关键词: 高职，男护生，手机使用，心理咨询，劳动教育 


\section{1. 前言}

随着 “生物一心理-社会” 医学模式的深入人心, 人们对护理职业有了进一步认识, 加之急诊科、手术 室、精神科、泌尿外科、ICU 病房、骨科等临床实践 的需要, 男护生的职业认同感和对口就业率越来越 高, 也使得越来越多的男生就读护理专业。但是在护 理专业的教室中和课堂上, 女生人数依然占优势, 在 传统理论或技能的授课方式下, 女生普遍擅长专注听 课、知识记忆、技能操作、师生互动等, 而为数不多 的男护生们, 在与女生学习效果的对比下, 越来越不 被老师关注, 学习的自信心被影响, 甚至逐渐被边缘 化, 越是对自己的评价低, 他们越会去手机网络中寻 求安慰 ${ }^{[1]}$, 于是他们上课时坐在教室的最后排, 使用 手机刷朋友圈、看短视频、聊天、玩游戏等不当行为 也随之越来越普遍化。长此以往, 男护生们的学习状 态和学习效果必然会越来越不理想, 同时他们的身体 健康也会受到不良影响, 因为过度使用手机会损伤正 中神经, 引起拇指疼痛, 甚至僵硬, 从而影响手部功 能; 同时长时间注视手机屏幕会对视力造成一定影 响, 表现为眼睛胀痛、视物模糊 ${ }^{[2]}$ 。

同时，手机使用的不当行为还会影响到其他方 面。自从互联网开始普及, 各种媒体就没有停止过对 由网络成瘾引发的各种事端的报道, 当前移动网络的 便利，又使得 “我在你身边，而你却在看手机” 的现 象比比皆是。选择读护理专业的高职男护生, 专业选 择的原因可能有学习能力较差、自控力较差、没有养 成良好的学习习惯、感情较丰富、心理易敏感脆弱、 社交能力欠缺等。在这个女性占明显优势的学习环境 中, 男护生们会更多地向网络的虚拟世界寻求寄托, 然而手机依赖, 又会影响他们正常的人际交往, 使得 他们更加内向、孤僻 ${ }^{[3]}$, 甚至自闭, 如此形成一个恶 性循环。

针对以上现象，高职学校的学生管理人员曾先后 采取过多种方法, 试图控制学生使用手机的时间, 例 如定时断电、断网; 教室悬挂手机袋, 上课时间手机 集中放置; 更有甚者, 一旦有管理者发现学生上课时 间使用手机, 会将其没收并限期集中存放等。但是这 些方法都不能从根本上改变学生使用手机的不当行 为, 他们总能想到应对的策略, 而且外力越大, 他们 压抑得越久, 反弹就会越剧烈, 可能会带来更多更严 重的问题。更何况手机和网络的便利, 已经惠及教学 实践, 教师上课使用蓝墨云班课等 APP 或微信小程序 等工具辅助教学, 已成为课堂改革的必然选择。尤其 是 2020 年以来, 新冠疫情让上网课成为教育的一种 常态, 如何管理好自己的时间和精力上好网课, 成为 从幼儿园小朋友到大学生都必备的能力。

所以, 一味地 “堵” 并不能解决问题, 如何引导 学生正确采取使用手机的行为, 才是正确的方向。对 于高职男护生的手机使用, 应进行有针对性的适度引 导, 而对其中部分已经出现网络依赖, 甚至成瘾的学 生，更应该进行耐心、细致地个性化辅导。

\section{2. 适度引导策略}

\section{1 开拓学生视野, 变手机为最便利的工具}

男护生们使用手机，大多局限在玩游戏、看视频、 聊天 ${ }^{[4]}$ 等各种娱乐功能，往往仅在需要完成作业的时 候才能想起用网络来查资料; 他们朋友圈里面分享的 以情感、美食、笑话、视频为主，偶尔出现一篇与所 学专业相关的文章, 最大的可能是某科考试的知识要 点; 他们平时习惯用手机拍照记录生活瞬间，却想不 起来在课堂上拍老师不断变化的板书、课件; 他们随 时会用手机的 “扫一扫” 功能扫描生活中随处可见的 二维码, 却偏偏不会扫描各科教材上附带的教学资 源。

针对这样的现状, 需要授课教师和学生管理人员 携起手来共同努力，多维度开展工作。授课教师可以 将一些生活或专业实践中，与所授科目相关的有趣 味、有助益的问题, 设计成作业的形式, 鼓励学生课 下上网查资料来自行解决, 然后在课堂上进行讨论分 享, 帮助学生逐渐养成上网查资料的习惯; 学生管理 人员可以注意收集各种适合学生阅读的小文, 包括与 情感相关的、提升人际交往能力的、关于阳光心态的, 以及一些与专业知识、职业发展有关的，发布在他们 方便看到的网络媒介上，鼓励他们阅读、评论、分享， 还要及时对评论做出反馈, 这样的互动有助于主流教 育占领学生思想的高地; 授课教师或学生管理人员都 可以选择一些有益的小程序或 APP 组织学生活动, 例 如微信小程序 “识花君”，既适合给生物类专业的学 生开展专业相关活动, 又适合给所有专业的学生开展 生活类的活动，结合适量劳动教育 ${ }^{[5]}$, 增长知识的同 时还可以有效实现身心健康; 学生的自觉学习习惯不 理想, 听课把握不住重点, 需要老师们适时地提醒他 们把重点内容拍照, 保存在他们自己手机里的图片, 增加了他们接触知识的机会与频率, 待学生们享受到 勤拍照勤记笔记的好处，就会自觉养成良好的习惯， 老师们也就不需要再多费口舌了。通过这样一系列的 方法, 我们才能够在网络这个汪洋大海中把握一定的 主动权, 让学生在潜移默化中学会更有益于身心健康 的手机使用行为。

\section{2 适度的 “行为疗法” 有助于学生重建良好 行为模式}

对于少部分使用手机已经严重到违反纪律的男 护生，他们会因为长时间玩手机导致不按时就寝，第 二天上课没精神，或者他们直接在上课时间玩手机， 这都会影响学习成绩, 也会影响课堂秩序, 这就需要 采用心理学上的 “行为疗法” 采取相应的惩罚措施, 来纠正他们的坏习惯。行为疗法的一个方面, 就是根 据学习理论或条件反射理论, 来矫正和消除不良条件 反射的行为。根据学生犯错的次数和程度, “量刑” 可以不同, 例如学生管理人员可以罚犯错学生多承担 班级事务、帮忙打扫办公室、跑腿上传下达文件材料 
等; 授课教师可以罚学生帮忙收作业、整理资料、修 订文本等。

说是惩罚, 实际上这些工作也有利于学生的成 长, 体力劳动的过程有辛苦又有乐趣, 现场人员反而 能够破除障碍深度交流, 增加学生之间和师生之间的 沟通; 跑腿送材料的过程能够让学生更多地了解学校 各个部门, 以及老师们的日常工作, 逐渐能够理解老 师，并学会换位思考; 整理、修订老师们需要的文字 资料，这项工作在帮任课老师减轻工作负担的同时， 还能让学生学到更多的知识, 而手机这个“娱乐设备” 也随之变成了自我提升的 “有力工具”。

惩罚不是目的, 通过惩罚能够让学生认识到自己 的错误、感受到老师们的关心关注, 不再有不当的使 用手机行为, 顺便还能让学生有些收获, 才符合 “情 感教育” ${ }^{[6]}$ 和 “因材施教” 的理念。

\section{3 不可忽视必要时的心理咨询}

对于极少数采用 “行为疗法” 收效甚微的学生, 他们身上各自存在着不同的问题, 或因为原生家庭娇 生惯养管教不严, 没有正确的自我定位, 缺乏前进的 动力与目标; 或是在成长过程中曾遭受挫折, 导致一 跉不振、自暴自弃; 或是有重大生活事件影响情绪, 正处于低谷时期; 或是对 “大学” 阶段的认识不清, 没有很好地适应大学生活; 或是被青春期末期的生理 因素影响, 对情感问题的关注过多, 不能保持足够的 精力在学习方面……总之, 因为种种原因, 他们会留 给老师们 “屡教不改” 且不服从管理的印象。

对于这些心理问题比较明显的学生, 他们在没有 得到理解之前, 甚至根本不愿配合老师的引导。这时, 老师们应采取的是更加个性化的方法, 尽量挖掘导致 他们手机使用不当行为的深层心理原因, 然后再有针 对性地采取干预措施。这可能需要多次的心理咨询才 能实现, 可能还需要周围同学和学生家长的支持与协 助。

\section{3. 一个多方联动的成功案例}

小晨是一名男护生，在他身上并没有前文述及的 那些男护生普遍存在的缺点, 相反, 他在本班三十多 名女护生和十几名男护生中, 表现一直是比较优秀 的。他入学后表现一直积极上进, 在班上被大家选为 班长, 在学生会是重点培养对象, 第一学期期末考试 成绩也很不错。然而第二学期开始后, 小晨的辅导员 发现, 一些交给他的事务在完成后, 是由其他班委向 辅导员进行反馈的, 学生会主席也反映小晨最近一段 时间表现不那么积极了, 有时会逃避一些日常事务之 外的工作, 但是同班同学们却反映他还是像以前一样 忙碌, 班上经常见不到他的影子。

\section{1 辅导员工作}

经辅导员的调查发现, 小晨是迷恋上了手游, 不 但上课时间会偷偷玩儿, 晚上謷夜玩儿影响正常起床 了, 还会以学生会有事为由逃课继续躲在宿舍玩儿, 周末回家也整天待在自己的房间里, 不再帮家里的小 超市工作。辅导员找机会把小晨叫到办公室谈话, 看 到他的精神状态大不如从前, 虽勉强掩饰还是显得很 疲劳。辅导员和颜悦色地用一些事实指出他的隐瞒, 他才肯承认最近上网比较多, 但继续争辩说并没有耽 误学习和工作。辅导员进行了一番摆事实讲道理, 用 同龄人的惨痛教训向他说明网络成瘾的危害, 提醒他 一定劳逸结合，管理好自己的时间，而小晨也给了辅 导员承诺不再继续沉迷。辅导员委托了几名同学, 对 小晨的行为观察了一段时间, 发现他还是没能有所控 制, 承诺只停留在了口头上。于是辅导员向小晨推荐 了学校的心理健康中心, 在小晨自愿接受心理咨询的 情况下, 辅导员帮他约见了一位有经验的心理咨询师 老师。

\section{2 心理咨询}

小晨与心理咨询师的谈话进行得比较顺利, 交谈 中他讲到了自己上网、玩手游的发展过程, 坦言高中 阶段就是因为自己管不住自己, 彻夜上网、打游戏, 导致高考失利, 只能来高职学校上学。上了大学本来 决心痛改前非, 好好施展才华, 有番作为, 但最近遇 到了一件事情又把自己带回了高考前那段灰暗的日 子。经过进一步工作, 小晨终于敞开心扉说到, 入学 后的出色表现让他广受好评, 许多女同学对他有好 感, 但他却只对一位从入学就带他接触学生会工作的 学姐心生爱慕, 一番追求不成, 反而使两人关系变得 冷淡, 这让他一踞不振, 重新投入网络游戏的怀抱。 这一段时间的沉迷让他十分后悔, 却又难以自控。后 来, 又通过 2 次心理咨询, 咨询师引导着小晨建立起 了正确的恋爱观, 帮助他能够明智地对待大学阶段的 感情问题, 并对他与异性交往的方式提出了建议。小 晨学会了用 “合理情绪理论” 与自己的不合理信念进 行辩论, 从而建立起了新的合理信念: 要把眼光放长 远, 现阶段的主要任务是为自己的人生有所实现而打 好基础, 未来的自己才能有资本经营自己的感情生 活; 即使现在在感情方面有所收获, 也要以二人互相 促进为前提, 若互拖后腿, 就只会留下无尽的遗憾, 最终也只会以感情失败告终。

\section{3 后续工作}

心理咨询结束时, 小晨已建立了坚定的信念, 要 改变自己无限制玩手游的不当行为, 他主动向辅导员 和同学们求助, 想获得大家的监督。于是, 辅导员对 他提出了具体要求，要当做完成作业一般，控制自己 拿起手机的次数和时间, 并要定期向辅导员汇报改善 进展。小晨又委托了同宿舍同学对自己进行监督、提 醒, 辅导员建议他对同学们这些善意的提醒要立即采 
取行动, 并要懂得感恩。后来, 小晨经常主动找辅导 员谈心，同宿舍同学也说他没有再长时间謷夜玩游 戏, 他还曾积极热情地向路遇的心理咨询师老师反映 自己的变化。期间, 小晨鼓起勇气和那名出色的学姐 进行了一番长谈, 了解了她对小晨的看法和她对自己 未来的规划, 这解开了小晨的心结, 两人现在不但可 以友好相处, 还可以互相倾诉心声。一个月后, 辅导 员再次与小晨的家长取得联系, 得知他在家也变得干 活多, 上网少了。另外, 因为知耻后勇, 在学生会改 选时, 小晨成功竞得了部长的职位。

\section{4 案例反思}

小晨手机使用不当行为问题的及时发现与介入， 辅导员、心理咨询师、宿舍同学、相关人物等, 用恰 当的心理与行为引导方法, 亲情、友情等良好的社会 支持系统, 多方联动采取有效措施, 最终使得小晨可 以悬崖勒马, 成功摆脱了手机使用不当行为。这个案 例成功的关键, 是教育工作者们的一份责任心, 使得 问题能够被及时发现、干预, 及时调动相关人员采取 有效措施, 并在事后注意跟踪效果反馈, 使得学生的 心理和行为问题可以获得持续改善。

\section{4. 结语}

在对手机使用不当的高职男护生群体进行干预 的过程中, 很难找到一种通用的方法可以适合所有学 生。教师可以精心设计一堂好课, 在课堂上让大多数 问题较轻微的学生受益, 然而剩下的小部分问题较重 的学生, 才是真正有挑战的, 会占用教师大量时间和 精力, 甚至生出 “恨铁不成钢” 的情感。教师们都应 化被动为主动, 积极引导学生使用手机服务于学习, 还应携起手来互相配合, 采用行为疗法、心理咨询等 多种方法因材施教。在与这小部分学生 “斗智斗勇” 的过程中, 教师们也需要不断充实自己, 获得自身的 提升, 这也许就是教育的鬼米力, 教师这个行业的价值。

\section{项目基金}

本文为以下基金项目的阶段性成果：1.滨州职业 学院教学改革研究项目《“学分+学点、动态项目、多
维同步、平台支撑” 新时代 “青马工程”培训班课程 体系构建研究》(编号: xyjg2122), 主持人: 谢根坦; 2.滨州职业学院科研项目《性别差异对高职生职业认 同感影响及对策的分析研究- - 以护理专业为例》

(编号：2017YJKT12)，主持人：毕智丽；3.滨州职 业学院科研项目《新时代高职劳动教育综合干预与质 量监测研究》(编号: 2020YJKT36), 主持人: 谢根 坦。

\section{作者简介:}

谢根坦（1980-)，男，河北冀州人，讲师，团 委书记, 研究方向为职业教育课程改革。

\section{REFERENCES}

[1] Xu, Z. F, Tang Y. Y. (2021) Analysis on the current situation and influencing factors of mobile phone dependence of higher vocational nursing students. Health Vocational Education, 39(10):132-135.

[2] Sahu, M, Gandhi, S, Sharma, M. K. (2019) Mobile phone addiction among children and adolescents: a systematic review. Journal of Addictions Nursing, 30(4):261-268.

[3] Wang, X. J, Xu, Y, Xu, B. L. (2020) Meta analysis of mobile phone addiction rate of nursing students in China. Psychological monthly, 15(21):38-39+85.

[4] Zhou, L. J. (2017) Investigation on mobile phone use behavior and mobile phone addiction of higher vocational nursing students. Contemporary Education Research and Teaching Practice (electronic journal), 8(9):993.

[5] Tan, C. B. (2019) Understanding the concept of labor education: how to understand the basic connotation and characteristics of labor education. Journal of the Chinese Society of Education, (02):82-84.

[6] Shen, X. Y. (2021) Analysis on the influence of emotional education on ideological and political education in colleges and universities. Journal of Jiamusi Vocational Institute, 37(10):15-16. 